\title{
Racial and Ethnic Differences Associated With Feeding- and Activity-Related Behaviors in Infants
}

AUTHORS: Eliana M. Perrin, MD, MPH, a,b Russell L. Rothman, MD, MPP, ${ }^{c}$ Lee M. Sanders, MD, MPH, ${ }^{d}$ Asheley C.

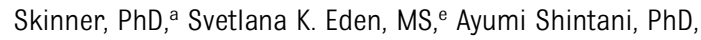

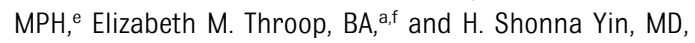
$\mathrm{MS}^{8}$

aDepartment of Pediatrics, School of Medicine, and ${ }^{b}$ Cecil G. Sheps Center for Health Services Research, University of North Carolina-Chapel Hill, Chapel Hill, North Carolina; Departments of ${ }^{c}$ Pediatrics, Internal Medicine, and ${ }^{~}$ Biostatistics, Vanderbilt University Medical Center, Nashville, Tennessee; 'Department of Pediatrics and Center for Health Policy, Stanford University,

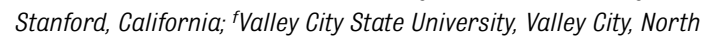
Dakota; and ${ }^{9}$ Department of Pediatrics, School of Medicine, New York University, New York, New York

\section{KEY WORDS}

obesity, infants, parenting, feeding, physical activity, television, breastfeeding

\section{ABBREVIATIONS}

AAP-American Academy of Pediatrics

aOR-adjusted odds ratio

$\mathrm{Cl}$-confidence interval

IFSQ - Infant Feeding Style Questionnaire

P0—-proportional odds

WIC-Supplemental Nutrition Program for Women, Infants, and Children

Dr Perrin helped conceptualize and design the study, helped design the data collection instruments, helped develop data collection protocols at all 4 sites, supervised data collection at 1 site, helped develop the analysis plan, drafted the initial manuscript, and reviewed and revised the manuscript according to feedback; Drs Rothman and Sanders helped conceptualize and design the study, helped design the data collection instruments, helped develop data collection protocols at all 4 sites, supervised data collection at 1 site, helped develop the analysis plan, and reviewed and revised the manuscript; $\mathrm{Dr}$ Skinner helped develop the analysis plan, helped analyze the data, helped draft the initial manuscript, and reviewed and revised the manuscript; Ms Eden helped develop the analysis plan, helped analyze the data, and reviewed and revised the manuscript; Dr Shintani supervised the development of the analysis plan, supervised the analysis of the data, and reviewed and revised the manuscript; Ms Throop supervised data collection at 1 site, and reviewed and revised the manuscript according to feedback; Dr Yin helped conceptualize and design the study, helped design the data collection instruments, helped develop data collection protocols at all 4 sites, supervised data collection at 1 site, helped develop the analysis plan, and reviewed and revised the manuscript; and all authors approved the final manuscript as submitted.

This trial has been registered at www.clinicaltrials.gov (identifier NCT01040897)

(Continued on last page)
WHAT'S KNOWN ON THIS SUBJECT: Although expert consensus and previous literature document the importance of early feeding and activity behaviors and practices in preventing obesity and the risks of early rapid weight gain, few studies have rigorously assessed obesity-related behaviors by caregivers of infants.

WHAT THIS STUDY ADDS: This study demonstrates the high prevalence of behaviors thought to increase risk for obesity in a diverse, large sample of parent/2-month-old dyads and finds that many behaviors vary by race and ethnicity, suggesting the potential for culturally tailored interventions.
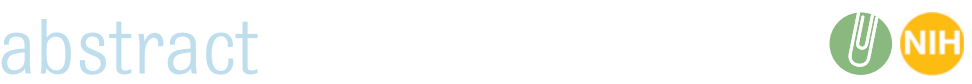

OBJECTIVE: To examine parental reports of feeding and activity behaviors in a cohort of parents of 2-month-olds and how they differ by race/ ethnicity.

METHODS: Parents participating in Greenlight, a cluster, randomized trial of obesity prevention at 4 health centers, were queried at enrollment about feeding and activity behaviors thought to increase obesity risk. Unadjusted associations between race/ethnicity and the outcomes of interest were performed by using Pearson $\chi^{2}$ and Kruskal-Wallis tests. Adjusted analyses were performed by using proportional odds logistic regressions.

RESULTS: Eight hundred sixty-three parents (50\% Hispanic, 27\% black, $18 \%$ white; $86 \%$ Medicaid) were enrolled. Exclusive formula feeding was more than twice as common (45\%) as exclusive breastfeeding (19\%); $12 \%$ had already introduced solid food; $43 \%$ put infants to bed with bottles; $23 \%$ propped bottles; $20 \%$ always fed when the infant cried; $38 \%$ always tried to get children to finish milk; $90 \%$ were exposed to television (mean, 346 minutes/day); $50 \%$ reported active television watching (mean, 25 minutes/day); and $66 \%$ did not meet "tummy time" recommendations. Compared with white parents, black parents were more likely to put children to bed with a bottle (adjusted odds ratio [a0R] $=1.97, P<.004$; bottle propping, a0R $=3.1$, $P<.001)$, and report more television watching $(\mathrm{a} 0 \mathrm{R}=1.6, P=.034)$. Hispanic parents were more likely than white parents to encourage children to finish feeding (aOR $=1.9, P=.007$ ), bottle propping (aOR = 2.5, $P=.009)$, and report less tummy time (aOR $=0.6, P=.037)$.

CONCLUSIONS: Behaviors thought to relate to later obesity were highly prevalent in this large, diverse sample and varied by race/ethnicity, suggesting the importance of early and culturally-adapted interventions. Pediatrics 2014;133:e857-e867 
Among 2-to 5-year-old US children, $27 \%$ are overweight or obese, with higher rates for Hispanic and non-Hispanic black children, ${ }^{1}$ illustrating the need for early obesity prevention and close examination for early racial/ethnic disparities in risk behaviors. Infancy may represent a critical period for obesity prevention..$^{2-4}$ Numerous studies ${ }^{5-9}$ demonstrate that infants with rapid weight gain in the first year of life or with higher weight status are more likely to be overweight later in life. ${ }^{5}$ A better understanding of the infant feeding and physical activity behaviors that may promote unnecessary weight gain, contextualized by ethnicity and culture, is critical to effective obesity prevention interventions.

Epidemiologic evidence from early infancy suggests that specific feeding content and feeding styles and practices may be related to obesity risk. Although there has been some controversy, ${ }^{10}$ increased duration and exclusivity of breastfeeding, for example, has been demonstrated to be generally protective against childhood obesity. ${ }^{11-15}$ In contrast, early introduction of solids ${ }^{16-20}$ and consumption of fruit juice and sweet drinks ${ }^{20-22}$ has been shown to be related to increased risk of obesity development.

Some studies have also suggested a relationship between early childhood obesity and caregiver feeding styles. ${ }^{23-26}$ Pressured feeding styles (eg, encouraging the infant to finish feeding) and "bottle propping" (ie, leaning a bottle up against a blanket instead of holding it) have been independently associated with increased obesity risk, whereas responsive feeding style (eg, feeding the infant in response to satiety cues) is associated with decreased risk. Some behaviors, like putting an infant to bed with a bottle, have only been related to obesity in older children. ${ }^{27}$ Similarly, the link between physical activity and obesity are well established during later childhood, ${ }^{28-33}$ yet less research has assessed the obesity risk associated with physical activity or sedentary activity (eg, screen time) during infancy. ${ }^{28-33}$

Ethnicity, race, and culture can play an important role in our identities and our health behaviors, including dietary and physical-activity behaviors that may potentiate obesity. Although these relationships are beginning to be understood among older children, ${ }^{34-37}$ we know less about the role of race/ ethnicity at the time when many of these behaviors are forged, namely the introduction of an infant into a family. We sought to examine the patterns of these behaviors theorized or known to have a relationship to later obesity in a diverse group of 2-monthold children and to assess how these patterns differed across parent-defined racial and ethnic groups.

\section{METHODS}

\section{Sites and Sample}

The analyses presented here are crosssectional baseline data reported by caregivers participating in the Greenlight Intervention Study. The Greenlight Study is a cluster randomized trial of an obesity prevention intervention targeting children during their first 2 years of life. Four university-affiliated pediatric clinics (University of North Carolina at Chapel Hill, New York University/ Bellevue Hospital Center, Vanderbilt University, and University of Miami/ Jackson Memorial Medical Center) are participating in the study, with 2 sites randomized to using a literacy and numeracy-sensitive approach to obesity prevention and 2 sites randomized to address injury prevention as an active control. We enrolled caregivers (mostly mothers and $\geq 18$ years old) of healthy infants at their infants' 2-month-old preventive services visit.
See Supplemental Information and Fig 1 for eligibility requirements and enrollment flow. Parents provided written and verbal consent according to institutional review board protocols approved at all 4 sites. Once consent was obtained, parents completed questionnaires with trained research assistants who were fluent in the language of parents' choice (English or Spanish). Data were managed through Research Electronic Data Capture, a secure, Webbased application designed to support data capture for research studies ${ }^{38}$ hosted at Vanderbilt University.

\section{Measures}

Previous literature on infant dietary and physical activity practices and relationship to obesity was reviewed $21,24,39-44$ to create questionnaires that were based, whenever possible, on previously validated measures of infant feeding behaviors, feeding styles such as questions derived from the Infant Feeding Style Questionnaire (IFSQ), and activity and screen time.

\section{Dietary Practices, Behaviors, and Attitudes}

Type of milk feeding, sweet drinks, early introduction of solids (including cereal in the bottle), and bottle-to-sleep behaviors were assessed. Parents were asked questions about how often they try to get their children to finish breast milk or formula, how often they watch television while feeding their infants, and how often they allow their infants to decide how much to eat. (See Supplemental Information for exact questions and response options.)

\section{Physical Activity Practices and Sedentary Time}

Physical activity practices such as "tummy time," television exposure (television on in the room the infant is in), and active television time (infant watching television) were assessed. In 


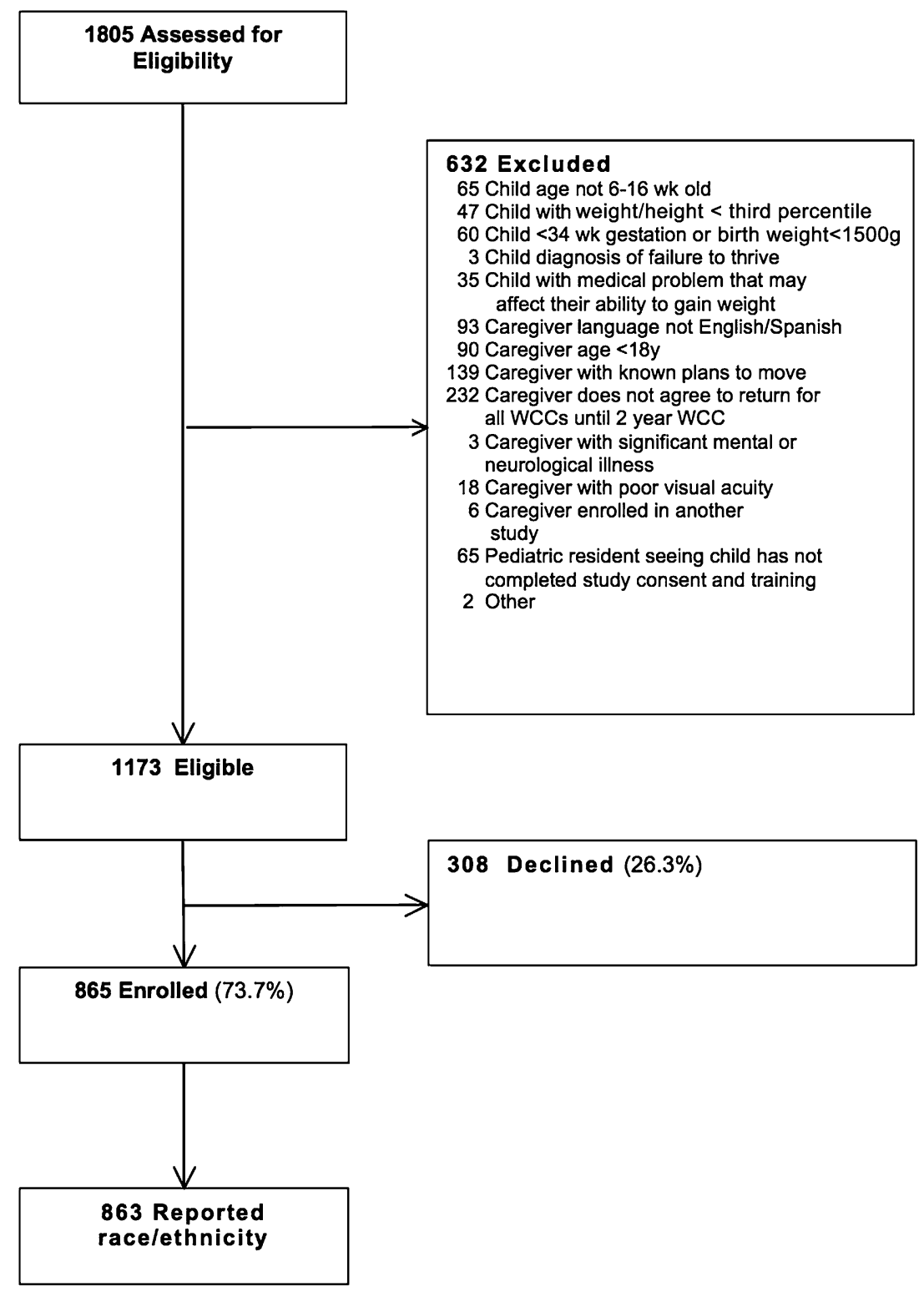

FIGURE 1

Eligibility requirements and enrollment flow. WCC, well child check.

addition to reporting mean values, we chose to dichotomize television watching as any compared with none based on the American Academy of Pediatrics (AAP) evidence-based practice guidelines that discourage children under the age of 2 years watching any television, 45 and we chose to dichotomize tummy time at $<$ versus $\geq 30$ minutes because of AAP recommendations to get 30 minutes of tummy time each day. ${ }^{46}$ See Supplemental Information for exact questions and response options.

\section{Independent Variables}

The primary independent variable of interest for this analysis was parent race or ethnicity assessed with 2 questions: "Do you consider yourself Hispanic/Latino?" and "What race do you consider yourself to be?" The response options were then categorized as Hispanic, white non-Hispanic, black non-Hispanic, or other non-Hispanic. We did not analyze race and ethnicity separately because the vast majority of individuals identifying as Hispanic did not also identify a race (eg, selected "other" and categorized themselves as "Hispanic" race).

\section{Statistical Analyses}

Patient's characteristics (age, gender, insurance) and respondent's characteristics (relationship to child, whether born in the United States, education, the language of survey completion, use of out of home child care, enrollment in the Supplemental Nutrition Program for Women, Infants, and Children [WIC], household income, and the number of children and adults in the home) were summarized by using mean and SD for continuous variables and proportions for categorical variables.

To assess the association between race/ethnicity and the outcomes of interest, unadjusted analyses were performed by using Pearson $\chi^{2}$ tests for all outcomes except active television watching, television exposure, and tummy time, for which Kruskal-Wallis tests were used. Adjusted analyses were performed by using proportional odds (PO) logistic regression. ${ }^{47}$ The PO model is a suitable and widely used tool for analyzing responses when there is a natural ordering of the values. For the purpose of this analysis, all outcomes with categorical responses were considered ordinal. P0 models were also used for continuous outcomes including active television watching, television exposure, and tummy time because the PO model works well also for continuous outcomes with skewed or sparse distributions. To prevent overfitting, ${ }^{48}$ all models were adjusted for the following a priori defined set of covariates: child gender, child out of home care (any, none), WIC status (yes, no), parent age, race/ethnicity (Hispanic, black non-Hispanic, other non-Hispanic, white non-Hispanic), language (Spanish, English), household income $(<\$ 10000$, \$10 000-\$19 999, \$20 000-\$39 999, 
$\$ 40000$ or more), number of adults in the home (1, 2, or more), number of children in the home (1, 2, or more), and study recruitment site (University of North Carolina at Chapel Hill, New York University/Bellevue Hospital Center, Vanderbilt University, and University of Miami/Jackson Memorial Medical Center). Parent age was modeled by using restricted cubic spline with 3 knots to detect if there was a nonlinear effect for age. ${ }^{48}$ For each outcome, a complete case analysis was performed. Outcome of sweet drinks was not analyzed by using an adjusted model because of too few patients with positive responses. Results were presented by using odds ratios comparing each race category to white race. The $P$ values and $95 \%$ confidence intervals (Cls) for odds ratios were calculated by using contrast. For each model, a global $P$ value was reported to indicate significance of race variable before examining the pairwise comparisons between race categories. Data were analyzed by using $R$ version 2.15 (www.r-project.org). For all analyses, a 2-tailed $P$ value of $<.05$ was considered statistically significant.

\section{RESULTS}

A total of 865 parents of infants met inclusion criteria, consented to the study, and were enrolled at baseline, and 863 reported race/ethnicity (Fig 1). Of these, the majority of parents selfidentified as Hispanic (49.8\%), followed by black non-Hispanic (27.3\%), white non-Hispanic (17.7\%), and other race, non-Hispanic (5.1\%). Across all sites, the largest Hispanic subgroup identified as North American/Mexican (55.7\%), followed by Central American (19.8\%), South American (14.5\%), Caribbean (8.9\%), and Peninsular (0.6\%). Most infants were insured by Medicaid (85.6\%) and lived in households with income under $\$ 40000$ per year (84\%). Use of WIC and family characteristics, including those that may influence infant-care behaviors, varied significantly by race/ethnicity (Table 1).

\section{Dietary Practices}

Table 2 shows behaviors for the complete sample and stratified by race and ethnicity. Type and exclusivity of feeding differed by race/ethnicity $(P<$ .001). Exclusive feeding with infant formula was more than twice as common $(45 \%)$ than was exclusive breastfeeding (19\%). Hispanic mothers were more likely to report any breastfeeding (69\%), compared with black mothers (33\%) and white mothers (46\%). Twelve percent of parents reported feeding their infants with solid food (primarily in the form of cereal in the bottle), and these practices were far more common among non-Hispanic black (24\%) and non-Hispanic white (16\%) families, when compared with Hispanic families (4\%). Only $3 \%$ of parents overall reported feeding their infants any sweet drinks (primarily juice).

\section{Feeding Styles and Practices}

Some feeding styles thought to relate to later obesity were fairly common in the sample (Table 2). Forty-three percent of parents reported putting their infant to bed with a bottle at least sometimes, and more than 1 in 5 (23\%) reported propping the bottle when feeding. Nearly half $(46 \%)$ watch television at least half the time they are feeding. Black non-Hispanic parents were more likely to put their child to bed with a bottle at least once a day, compared with white non-Hispanic or Hispanic parents (37\% vs $22 \%)$. Hispanic parents were more likely (69\%) than black non-Hispanic parents (50\%) and white non-Hispanic parents (29\%) to encourage the infant to finish the bottle with almost every feed. Similarly, Hispanic parents were more likely (47\%) than black non-Hispanic parents (19\%) and white non-Hispanic parents (16\%) to immediately feed their infant with crying. Black non-Hispanic parents were more likely to ever prop their child's bottle $(35 \%)$, compared with Hispanic parents (20\%) and white nonHispanic parents (15\%). Finally, Hispanic parents were less likely to regularly (more than half the time) watch television while feeding $(37 \%)$ than white non-Hispanic parents $(53 \%)$ or black parents $(61 \%)$.

Physical Activity, Screen Time, and Sedentary Behaviors

Approximately half of all parents reported that their infants actively watched television, with an average of 25 minutes a day. Active television viewing also varied by race/ethnicity $(P<.001)$ and was lowest among Hispanic infants (41\%) and highest among non-Hispanic black infants (68\%). Additionally, over $90 \%$ of all infants were exposed to television, which also varied by race/ethnicity $(P<.001)$ with an average of 346 minutes per day (median of 180 minutes per day). Exposure was greatest among black non-Hispanic infants, with an average of 546 minutes per day, and least among Hispanic infants, with an average of 228 minutes/day. Approximately one-third (34\%) of all parents reported that their infants were given at least 30 minutes daily of tummy time. Hispanic infants were least likely to exceed this threshold (22\%), whereas black and white infants were more likely ( $45 \%$ and $46 \%$, respectively).

\section{Adjusted Analyses}

In the adjusted analysis (Table 3), race/ ethnicity characteristics remained independently associated with all behaviors thought to promote or protect against obesity, except for tummy time $(P<$ .05 , joint tests for all race/ethnicity categories). Compared with white non-Hispanic parents, black parents (adjusted odds ratio $[\mathrm{aOR}]=1.97 ; 95 \%$ 
TABLE 1 Characteristics of Study Population by Race/Ethnicity

\begin{tabular}{|c|c|c|c|c|c|c|}
\hline & \multirow{2}{*}{$\begin{array}{c}\text { Total } \\
(N=863)^{\mathrm{a}}\end{array}$} & \multicolumn{4}{|c|}{ Race/Ethnicity } & \multirow[t]{2}{*}{$P$} \\
\hline & & Hispanic $(N=430)$ & White $(N=153)$ & Black $(N=236)$ & Other $(N=44)$ & \\
\hline \multicolumn{7}{|l|}{ Child characteristics } \\
\hline Age, mo, mean (SD) & $2.1(0.4)$ & $2.1(0.4)$ & $2.1(0.4)$ & $2.2(0.4)$ & $2.1(0.3)$ & .4 \\
\hline Gender, girl & $443(51.3)$ & $214(49.8)$ & 76 (49.7) & $129(54.7)$ & $24(54.5)$ & .6 \\
\hline Out of home child care, any ${ }^{\mathrm{b}}$ & $79(9.2)$ & $26(6.1)$ & $18(12.0)$ & $34(14.4)$ & $1(2.3)$ & $<.001$ \\
\hline WIC status, ${ }^{\mathrm{C}}$ any & 729 (85.2) & 389 (90.9) & $96(64.0)$ & $216(92.3)$ & $28(63.6)$ & $<.001$ \\
\hline Insurance $^{d}$ & & & & & & $<.001$ \\
\hline Medicaid & 735 (85.6) & $381(88.8)$ & $110(73.3)$ & $215(91.1)$ & $29(65.9)$ & - \\
\hline Private (any) & $97(11.3)$ & $31(7.2)$ & $35(23.3)$ & $19(7.6)$ & $13(29.5)$ & - \\
\hline None & $27(3.1)$ & $17(4.0)$ & $5(3.3)$ & $3(1.3)$ & $2(4.5)$ & - \\
\hline \multicolumn{7}{|l|}{ Parent characteristics } \\
\hline Age, ${ }^{e}$ years, mean (SD) & $27.6(6.2)$ & $28.2(5.9)$ & $26.7(5.7)$ & $26.6(6.3)$ & $30.0(6.4)$ & $<.001$ \\
\hline Relationship to child & & & & & & .3 \\
\hline Mother & $825(95.6)$ & $413(96.0)$ & $148(96.7)$ & $226(95.8)$ & $38(86.4)$ & - \\
\hline Father & $36(4.2)$ & $16(3.7)$ & $5(3.3)$ & $9(3.8)$ & $6(13)$ & - \\
\hline Other & $2(0.2)$ & $1(0.2)$ & $0(0.0)$ & $1(0.4)$ & $0(0.0)$ & - \\
\hline Non-US born ${ }^{b}$ & $438(51.0)$ & $356(83.0)$ & $21(14.0)$ & $31(13.1)$ & $30(68.2)$ & $<.001$ \\
\hline Race/ethnicity ${ }^{\mathrm{f}}$ & & - & - & - & - & - \\
\hline Hispanic & $430(49.8)$ & - & - & - & - & - \\
\hline White, non-Hispanic & $153(17.7)$ & - & - & - & - & - \\
\hline Black, non-Hispanic & $236(27.3)$ & - & - & - & - & - \\
\hline Other, non-Hispanic & $44(5.1)$ & - & - & - & - & - \\
\hline Spanish language $e^{g}$ & $302(35.1)$ & $302(70.6)$ & $0(0.0)$ & $0(0.0)$ & $0(0.0)$ & $<.001$ \\
\hline Education ${ }^{\mathrm{h}}$ & & & & & & $<.001$ \\
\hline Less than high school & $225(26.2)$ & $176(40.0)$ & $13(8.7)$ & $35(14.9)$ & $1(2.3)$ & - \\
\hline HS graduate/General Educational Development & $280(32.6)$ & $129(30.1)$ & $44(29.3)$ & $101(43.0)$ & $6(13.6)$ & - \\
\hline Some college & $200(23.3)$ & $68(15.9)$ & $51(34.0)$ & $66(28.1)$ & $15(34.1)$ & - \\
\hline College or greater & $153(17.8)$ & $56(13.1)$ & $42(28.0)$ & $33(14.0)$ & $22(50.0)$ & - \\
\hline \multicolumn{7}{|l|}{ Household characteristics } \\
\hline Household income ${ }^{i}$ & & & & & & $<.001$ \\
\hline$<\$ 10000$ & $265(32.0)$ & $149(35.9)$ & $18(12.4)$ & $86(38.4)$ & $12(27.9)$ & - \\
\hline$\$ 10000-19999$ & $228(27.6)$ & $133(32.0)$ & $32(22.1)$ & $59(26.3)$ & $4(9.3)$ & - \\
\hline$\$ 20000-39999$ & $202(24.4)$ & $89(21.4)$ & $46(31.7)$ & $57(25.4)$ & $10(23.3)$ & - \\
\hline$\$ 40000$ or more & $132(16.0)$ & $44(10.6)$ & $49(33.8)$ & $22(9.8)$ & $17(39.5)$ & - \\
\hline Children in household ${ }^{\mathrm{b}}(<18$ years) & & & & & & $<.001$ \\
\hline 1 child & $342(39.8)$ & $149(34.7)$ & $77(51.3)$ & $89(37.7)$ & $27(61.4)$ & - \\
\hline 2 or more children & $517(60.2)$ & $280(65.3)$ & $73(48.7)$ & $147(62.3)$ & $17(38.6)$ & - \\
\hline \multicolumn{7}{|l|}{ Site characteristics } \\
\hline Site name & & & & & & $<.001$ \\
\hline University of Miami & $149(17.3)$ & $75(17.4)$ & $7(4.6)$ & $55(23.3)$ & $12(27.3)$ & - \\
\hline University of North Carolina- Chapel Hill & $255(29.5)$ & $97(22.6)$ & $61(39.9)$ & $85(36.0)$ & $12(27.3)$ & - \\
\hline Vanderbilt University & $230(26.7)$ & $78(18.1)$ & $70(45.8)$ & $74(31.4)$ & $8(18.2)$ & - \\
\hline New York University & $229(26.5)$ & $180(41.9)$ & $15(9.8)$ & $22(9.3)$ & $12(27.3)$ & - \\
\hline
\end{tabular}

Data presented as $N$ (\%) except as noted.

a After accounting for 2 subjects missing race/ethnicity.

${ }^{\mathrm{b}}$ Missing for 7 subjects.

${ }^{\mathrm{C}}$ Child or mother participating in WIC program; missing for 10 subjects.

${ }^{d}$ Child with any private insurance categorized as private; missing for 7 subjects.

e Missing for 6 subjects.

${ }^{\mathrm{f}}$ Missing for 3 subjects.

s Parent answered Spanish questionnaires as opposed to English questionnaires.

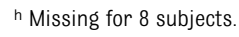

i Missing for 39 subjects.

$\mathrm{Cl}: 1.25-3.12)$ and other race parents $(\mathrm{a} 0 \mathrm{R}=2.26 ; 95 \% \mathrm{Cl}: 1.12-4.57)$ were more likely to put their child to bed with a bottle. Hispanic parents $(\mathrm{aOR}=$ 1.93; $95 \% \mathrm{Cl}: 1.20-3.11)$ and black parents (a0R $=1.62 ; 95 \% \mathrm{Cl}: 1.09-2.41)$ were more likely to encourage their child to finish breast milk or formula. Hispanic parents $(\mathrm{aOR}=2.47 ; 95 \% \mathrm{Cl}$ : 1.25-4.88) and black non-Hispanic parents $(\mathrm{aOR}=3.05 ; 95 \% \mathrm{Cl}: 1.71-$ 5.43) were also more likely to bottle prop. Parents who self-identified as non-Hispanic other race were more likely to feed their child immediately with crying $(\mathrm{aOR}=3.08$; $95 \% \mathrm{Cl}$ : $1.65-$ 5.74) and less likely to report television viewing when feeding ( $\mathrm{aOR}=0.46$; $95 \%$ 
TABLE 2 Eating and Activity Behaviors by Race/Ethnicity

\begin{tabular}{|c|c|c|c|c|c|c|}
\hline & \multirow{2}{*}{$\begin{array}{c}\text { Total } \\
(N=863)\end{array}$} & \multicolumn{4}{|c|}{ Race/Ethnicity } & \multirow[t]{2}{*}{$P$} \\
\hline & & Hispanic $(N=430)$ & White $(N=153)$ & Black $(N=236)$ & Other $(N=44)$ & \\
\hline \multicolumn{7}{|l|}{ Diet-Related Practices } \\
\hline Type of feeding & & & & & & $<.001$ \\
\hline Formula only & 385 (44.6) & $133(30.9)$ & $83(54.2)$ & $157(66.5)$ & $12(27.3)$ & - \\
\hline Mostly formula, and some breast milk & $127(14.7)$ & $82(19.1)$ & $10(6.5)$ & $25(10.6)$ & $10(22.7)$ & - \\
\hline Equal formula and breast milk & $11(1.3)$ & $9(2.1)$ & $0(0.0)$ & $2(0.8)$ & $0(0.0)$ & - \\
\hline Mostly breast milk, and some formula & $175(20.3)$ & $120(27.9)$ & 18 (11.8) & $29(12.3)$ & $8(18.2)$ & - \\
\hline Breast milk only & $165(19.1)$ & $86(20.0)$ & $42(27.5)$ & $23(9.7)$ & $14(31.8)$ & - \\
\hline Sweet drinks & & & & & & .9 \\
\hline Any & $26(3.0)$ & $12(2.8)$ & $6(3.9)$ & $7(3.0)$ & $1(2.3)$ & - \\
\hline None & $837(97.0)$ & $418(97.2)$ & $147(96.1)$ & $229(97.0)$ & $43(97.7)$ & - \\
\hline Early introduction of solids & & & & & & $<.001$ \\
\hline Yes & 98 (11.6) & $16(3.8)$ & 24 (15.9) & $55(23.5)$ & $3(7.1)$ & - \\
\hline No & $748(88.4)$ & $403(96.2)$ & $127(84.1)$ & $179(76.5)$ & 39 (92.9) & - \\
\hline \multicolumn{7}{|l|}{ Infant feeding style and practices } \\
\hline $\begin{array}{l}\text { In last } 2 \text { wk, how often was the infant } \\
\text { put to sleep while drinking a bottle }{ }^{a}\end{array}$ & & & & & & $<.001$ \\
\hline At most sleep times & $108(12.5)$ & $51(11.9)$ & $18(11.8)$ & $32(13.7)$ & $7(15.9)$ & - \\
\hline Sometimes, at least once a day & $119(13.8)$ & $41(9.5)$ & $15(9.8)$ & $54(23.1)$ & $9(20.5)$ & - \\
\hline Sometimes, but not every day & $143(16.6)$ & $80(18.6)$ & $18(11.8)$ & $41(17.5)$ & $4(9.1)$ & - \\
\hline Never & $491(57.0)$ & $258(60.0)$ & $102(66.7)$ & $107(45.7)$ & $24(54.5)$ & - \\
\hline $\begin{array}{l}\text { I try to get my child to finish her } \\
\text { breast milk or formula }\end{array}$ & & & & & & $<.001$ \\
\hline Always & $324(37.6)$ & $223(51.9)$ & $21(13.8)$ & $70(29.7)$ & $10(22.7)$ & - \\
\hline Most of the time & $149(17.3)$ & $73(17.0)$ & $23(15.1)$ & 47 (19.9) & $6(13.6)$ & - \\
\hline Half of the time & $75(8.7)$ & $21(4.9)$ & 19 (12.5) & $31(13.1)$ & $4(9.1)$ & - \\
\hline Seldom or infrequently & $103(11.9)$ & $37(8.6)$ & $37(24.3)$ & $25(10.6)$ & $4(9.1)$ & - \\
\hline Never & $211(24.5)$ & $76(17.7)$ & $52(34.2)$ & $63(26.7)$ & $20(45.5)$ & - \\
\hline $\begin{array}{l}\text { When my infant cries, I immediately } \\
\text { feed him or her }{ }^{a}\end{array}$ & & & & & & $<.001$ \\
\hline Always & $175(20.3)$ & $133(31.0)$ & $9(5.9)$ & $22(9.3)$ & $11(25.0)$ & - \\
\hline Most of the time & $119(13.8)$ & $67(15.6)$ & $16(10.5)$ & $23(9.7)$ & $13(29.5)$ & - \\
\hline Half of the time & $184(21.4)$ & 68 (15.9) & $42(27.6)$ & $65(27.5)$ & $9(20.5)$ & - \\
\hline Seldom or infrequently & $176(20.4)$ & $86(20.0)$ & $32(21.1)$ & $52(22.0)$ & $6(13.6)$ & - \\
\hline Never & $207(24.0)$ & $75(17.5)$ & $53(34.9)$ & $74(31.4)$ & $5(11.4)$ & - \\
\hline $\begin{array}{l}\text { When my child has a bottle, } \\
\text { I prop it up }{ }^{c}\end{array}$ & & & & & & $<0.001$ \\
\hline Always & $31(3.7)$ & $16(3.9)$ & $0(0.0)$ & $13(5.6)$ & $2(4.8)$ & - \\
\hline Most of the time & $24(2.9)$ & $9(2.2)$ & $3(2.0)$ & $11(4.7)$ & $1(2.4)$ & - \\
\hline Half of the time & $37(4.4)$ & $13(3.2)$ & $3(2.0)$ & $21(9.0)$ & $0(0.0)$ & - \\
\hline Seldom or infrequently & $102(12.2)$ & $43(10.4)$ & $17(11.3)$ & $36(15.5)$ & $6(14.3)$ & - \\
\hline Never & $643(76.8)$ & $331(80.3)$ & $127(84.7)$ & $152(65.2)$ & $33(78.6)$ & - \\
\hline $\begin{array}{l}\text { I watch television while feeding } \\
\text { my infant }\end{array}$ & & & & & & $<0.001$ \\
\hline Always & $72(8.4)$ & $34(7.9)$ & $10(6.6)$ & $25(10.6)$ & $3(6.8)$ & - \\
\hline Most of the time & $101(11.7)$ & $34(7.9)$ & $22(14.5)$ & $44(18.6)$ & $1(2.3)$ & - \\
\hline Half of the time & 223 (25.9) & $91(21.2)$ & $49(32.2)$ & 75 (31.8) & $8(18.2)$ & - \\
\hline Seldom or infrequently & $252(29.2)$ & $140(32.6)$ & $48(31.6)$ & 47 (19.9) & $17(38.6)$ & - \\
\hline Never & $214(24.8)$ & $131(30.5)$ & $23(15.1)$ & $45(19.1)$ & $15(34.1)$ & - \\
\hline \multicolumn{7}{|l|}{ Physical activity-related practices } \\
\hline Tummy time, minutes, mean (SD) & $37.5(85.4)$ & $26.2(80.1)$ & $44.9(63.0)$ & $52.2(104.6)$ & $44.2(77.3)$ & $<.001$ \\
\hline Tummy time $^{d}$ & & & & & & $<.001$ \\
\hline 30 min or more & $288(33.5)$ & $94(22.0)$ & $70(46.1)$ & $107(45.3)$ & $17(39.5)$ & - \\
\hline$<30 \min$ & $571(66.5)$ & $334(78.0)$ & $82(53.9)$ & $129(54.7)$ & $26(60.5)$ & - \\
\hline Television exposure, ${ }^{a}$ min, mean (SD) & $346(395)$ & $228(266)$ & $397(401)$ & $546(494)$ & $267(385)$ & $<.001$ \\
\hline Television watching, ${ }^{\mathrm{a}}$ min mean (SD) & $25(66)$ & $11(31)$ & $24(45)$ & $51(107)$ & $16(45)$ & $<.001$ \\
\hline
\end{tabular}


TABLE 2 Continued

\begin{tabular}{|c|c|c|c|c|c|c|}
\hline & \multirow{2}{*}{$\begin{array}{c}\text { Total } \\
(N=863)\end{array}$} & \multicolumn{4}{|c|}{ Race/Ethnicity } & \multirow[t]{2}{*}{$P$} \\
\hline & & Hispanic $(N=430)$ & White $(N=153)$ & Black $(N=236)$ & Other $(N=44)$ & \\
\hline Television watching ${ }^{\mathrm{a}}$ & & & & & & $<.001$ \\
\hline Any & $429(49.8)$ & $174(40.6)$ & $78(51.3)$ & $161(68.2)$ & $16(36.4)$ & - \\
\hline None & $432(50.2)$ & $255(59.4)$ & 74 (48.7) & 75 (31.8) & $28(63.6)$ & - \\
\hline
\end{tabular}

Data presented as $N$ (\%) except as noted.

a Missing for 2 subjects.

${ }^{b}$ Missing for 1 subject.

c Missing for 26 subjects.

d Missing for 4 subjects.

Cl: 0.24-0.87). Black parents reported more frequent television watching for their infants $(\mathrm{aOR}=1.58 ; 95 \% \mathrm{Cl}$ : 1.04-2.41) than white non-Hispanic parents.

\section{DISCUSSION}

In this multisite, large sample of geographically, racially, and ethnically diverse, low-income parents caring for 2-month-old infants, we discovered a high frequency of parent-reported behaviors that may increase their children's risk for obesity. Even after adjusting for potential confounders, including parent age and socioeconomic status, self-reported race and ethnicity were strongly and independently associated with many of these behaviors thought to relate to later obesity.

This study is one of the first in the United States to report such behaviors so early in infancy. We found that only $34 \%$ of parents adhere to the AAPrecommended 30 minutes or more of daily tummy time ${ }^{46}$ In addition, average television exposure for these infants was over 5 hours per day, and, perhaps more striking, half of all parents expose their infant to "active TV viewing" for an average of more than 25 minutes per day. Already by 2 months, only $19 \%$ received breast milk exclusively, $38 \%$ were encouraged to finish a full bottle of formula or breast milk with every feeding, $23 \%$ had bottles propped in their crib or bassinet, and $12 \%$ were fed solid food.

Our findings add to a portrait of studies documenting eating and activity behaviors in the families of young children, 37 including ethnic disparities in these behaviors that some have argued may contribute significantly to the higher risk of obesity-related disease in ethnic minority communities. ${ }^{49}$ If these behaviors are truly "obesogenic," however, families from all races and ethnicities studied need early counseling, and the findings here also underscore the likely need for culturally sensitive health behavior counseling during early infancy. Particularly actionable are the specific behaviors that may be most sensitive to culturally adapted interventions: (1) infant exposure to television and other visual media; (2) breastfeeding initiation and exclusivity; and (3) encouraging infants to finish bottles. Further research, using mixed method approaches that apply quantitative and qualitative techniques, may help elucidate whether cultural differences play a larger role than family history or other concerns arising from individual family behaviors that put children at high risk for cardiovascular disease and diabetes.

Although previous studies have revealed high rates of young children watching television, ${ }^{50-52}$ our study results reveal surprisingly high rates of 2-month-old infants exposed to active television viewing and feeding while watching television, which may decrease responsive feeding. Meanwhile, although the AAP discourages television before age 2 years, ${ }^{45}$ only $15 \%$ of parents report their pediatrician discussed media use with them. ${ }^{51}$ These results suggest providers should begin counseling about screen time much earlier than is currently practiced, perhaps as part of routine newborn care.

As other studies have observed, ${ }^{37,53,54}$ we found significant and complex ethnic

TABLE 3 PO Logistic Regression for Feeding and Activity Behaviors; White Non-Hispanic Is Referent

\begin{tabular}{lccc}
\hline Outcome, $N$, Overall $P$ for Race/Ethnicity & Hispanic, aOR (95\% Cl), $P$ & Black, non-Hispanic, a0R (95\% Cl), $P$ & Other, non-Hispanic, a0R (95\% Cl), $P$ \\
\hline More formula than breast milk, 820, .029 & $0.70(0.43-1.17), .174$ & $1.31(0.82-2.10), .261$ & $0.65(0.33-1.26), .199$ \\
Bottle to bed, 819, .003 & $1.05(0.61-1.78), .867$ & $1.97(1.25-3.12), .004$ & $2.26(1.12-4.57), .023$ \\
Finish breast milk/formula, 820, .012 & $1.93(1.20-3.11), .007$ & $1.62(1.09-2.41), .018$ & $0.88(0.46-1.68), .698$ \\
Immediately feed, $819, .003$ & $1.24(0.78-1.96), .362$ & $1.05(0.70-1.57), .821$ & $3.08(1.65-5.74),<.001$ \\
Bottle propping, 797, .002 & $2.47(1.25-4.88), .009$ & $3.05(1.71-5.43),<.001$ & $2.26(0.92-5.58), .076$ \\
Television while feeding, 820, .014 & $1.04(0.66-1.63), .872$ & $1.29(0.87-1.92), .213$ & $0.46(0.24-0.86), .015$ \\
Active television watching, 819, .006 & $0.88(0.53-1.43), .595$ & $1.58(1.04-2.41), .034$ & $0.57(0.28-1.19), .133$ \\
Tummy time, 817, .083 & $0.62(0.40-0.97), .037$ & $0.86(0.58-1.28), .447$ & $0.53(0.28-1.003), .051$ \\
\hline
\end{tabular}

Outcomes are 5-point categorical variables for feeding behaviors; outcomes are continuous minutes of television watching and tummy time. 
disparities in the patterns of infant exposure to potentially unhealthy behaviors. Although this study has identified important differences in infant feeding and activity behaviors by race/ ethnicity, caution is needed when applying these findings to future clinical intervention. Because we do not yet know which of these behaviors are definitively linked to future obesity risk, we must be circumspect about designing any interventions based on racial, ethnic, or socioeconomic differences in the expression of these behaviors. Infants of Hispanic parents were protected from many behaviors thought to be related to later obesity. Compared with non-Hispanic parents, Hispanic parents were less likely to report exclusive formula feeding, less likely to report feeding solids, and less likely to report active and passive television viewing. However, Hispanic parents, compared with white parents, were more likely to report encouraging their infant to finish milk and bottle propping, and less likely to report providing their infants with as much tummy time each day. By contrast, although infants of black parents engaged in more daily tummy time, they were more likely to report some behaviors thought to be related to future obesity such as exclusive formula feeding, giving infants bottles to sleep, introducing solid foods, and watching television. Multiple explanations for these differences can be hypothesized and explained in part by their historical and environmental contexts. ${ }^{55}$ In 1 study, for example, reasons cited for not breastfeeding varied by race/ ethnicity, with Hispanic mothers less likely to cite household responsibilities as a barrier than black or white mothers. ${ }^{56}$ And the high rates of television for infants of black mothers may be related to their own higher rates of television watching. ${ }^{57}$ Clearly further qualitative research is needed to determine the relationships between culture and health behaviors and whether behaviors are modifiable through culturally tailored early intervention.

Although this study included a large, diverse sample, there are limitations to consider. First, there are difficulties and variability in defining race and ethnicity in research. ${ }^{58}$ As the Institute of Medicine argues, ${ }^{59}$ race is a social and cultural context that can be difficult to align on a single dimension. Differences within subgroups likely come into play in a way that was not analyzed here. For example, discrepancies of behavior that relate to country or region of origin for those who identified as "Hispanic" or even white were masked by a broad stroke identification of that grouping within this study. Furthermore, the act of using "white" as the referent population is problematic both symbolically and because white children also have high rates of obesity. Doing so also likely hides any subgroup differences. Different subgroups of the various races and ethnicities may relate to differences in cultural practices of the behaviors measured. These differences obviously deserve further study, potentially through qualitative work.

Second, the relationships we note are clearly related to underlying community and familial environments, including confounding biases unmeasured in our study that may together result in "cumulative social adversity" found to be related to childhood obesity. 60 Furthermore, social desirability bias is often associated with self-reported behaviors, which means both that the prevalence of specific behaviors may be underestimated and the relative prevalence of these behaviors in different ethnic groups may be influenced by differential desirability bias across these groups. Although we modeled behaviors thought to be related to obesity according to previous literature, no previously validated scale for several behaviors (including television "use") was available at the time of the study for parents of such young infants, and some of the behaviors we looked at (such as tummy time) have not had documented relationships to obesity and were only theorized to be important. Certainly a validated measure would have lent additional rigor to our study. Although we have included several clinically relevant questions from the validated IFSQ, ${ }^{24}$ we did not use the whole scale or complete subscales for specific feeding styles because of the respondent burden incurred from the rest of the intervention's measures. Although the cohort is diverse, it is not nationally representative, so generalizability may be limited. Finally, although this study presents baseline data from a longitudinal cohort, its findings are crosssectional, impairing any ability to infer causality. In future analyses, we hope to report on the relationship between early parent infant care behaviors and later indicators of child health, including weight status.

\section{CONCLUSIONS}

Our findings add further texture to the conceptual models and actionable recommendations of several national efforts to address the nation's obesity epidemic. The consensus Expert Panel, convened in 2008 by Shaping America's Health and The Obesity Society, encouraged the use of a socioecological framework ${ }^{61}$ to understand and address ethnic disparities in obesity and related behaviors. This model "views children in the context of their families, communities, and cultures, emphasizing the relationships among environmental, biological, and behavioral determinants of health" and "focuses on interactions between a person's physical, social, and cultural surroundings." Efforts to attenuate health disparities must attend to cultural 
patterns of dietary and physical-activity behaviors that are often established and reinforced during infancy. A population-wide child health problem like obesity demands a populationbased understanding of healthy behaviors of these children's environments: not merely at the level of ethnic and racial identity of their parents, but more importantly at the levels of the communities, neighborhoods, and families in which they live. Although office-based approaches to preventive care remain important, our results suggest that public health approaches should also account for the distinct cultural differences in

\section{REFERENCES}

1. Ogden CL, Carroll MD, Kit BK, Flegal KM. Prevalence of obesity and trends in body mass index among US children and adolescents, 1999-2010. JAMA. 2012;307(5): 483-490

2. Gillman MW. The first months of life: a critical period for development of obesity. Am J Clin Nutr. 2008;87 (6):1587-1589

3. Leunissen RW, Kerkhof GF, Stijnen T, Hokken-Koelega A. Timing and tempo of first-year rapid growth in relation to cardiovascular and metabolic risk profile in early adulthood. JAMA. 2009;301(21): 2234-2242

4. Taveras EM, Rifas-Shiman SL, Sherry B, et al. Crossing growth percentiles in infancy and risk of obesity in childhood. Arch Pediatr Adolesc Med. 2011;165(11):993-998

5. Baird J, Fisher D, Lucas P, Kleijnen J, Roberts $\mathrm{H}$, Law $\mathrm{C}$. Being big or growing fast: systematic review of size and growth in infancy and later obesity. BMJ. 2005;331 (7522):929-931

6. Lindberg SM, Adams AK, Prince RJ. Early predictors of obesity and cardiovascular risk among American Indian children. Matern Child Health J. 2012;16(9):18791886

7. Dubois L, Girard M. Early determinants of overweight at 4.5 years in a populationbased longitudinal study. Int $J$ Obes (Lond). 2006;30(4):610-617

8. Taveras EM, Rifas-Shiman SL, Belfort MB, Kleinman KP, Oken E, Gillman MW. Weight status in the first 6 months of life and a community's collective behaviors. Through culturally relevant, low-literacy and low-numeracy counseling and materials that target these behaviors, the Greenlight intervention may help reduce the prevalence of such behaviors and even obesity itself at age 2 years.

\section{ACKNOWLEDGMENTS}

We thank our colleagues Anna Maria Patino Fernandez, PhD, Alan Mendelsohn, MD, Benard Dreyer, MD, and Alan Delamater, PhD, for their assistance with the study. We thank Carol Runyan, PhD, Tamera Coyne-Beasley, MD, Shari Barkin, MD, MHS, and Judy Schaechter,
$M D$, for their expert assistance with measures, as well as our research coordinators Andrea J. Bacchus, BA, Joanne Finkle, JD, RN, Sophie Ravanbakht, BA Lucila Bloise, BA, Maria Cerra, BA, Evelyn Cruzatte, BA, and Daniela Quesada, BA, MPH. We thank our clinic champions, Michael Steiner, MD, MPH, Tamera Coyne-Beasley, MD, MPH, Cindy 0sman, MD, Steve Paik, MD, MSEd, Mary Jo Messito, MD, Barron Patterson, MD, and Seth Scholer, MD, MPH. Finally, we thank Rui Jiang, BA, Camilla Peterson, BSc, Janna Howard, BA, Brenda Calderon, $B A$, and our entire research staff at all sites for their assistance in this study. obesity at 3 years of age. Pediatrics. 2009; 123(4):1177-1183

9. Wright CM, Cox KM, Sherriff A, FrancoVilloria M, Pearce MS, Adamson AJ; Gateshead Millennium Study Core Team. To what extent do weight gain and eating avidity during infancy predict later adiposity? Public Health Nutr. 2012;15(4): 656-662

10. Martin RM, Patel R, Kramer MS, et al. Effects of promoting longer term and exclusive breastfeeding on cardiometabolic risk factors at age 11.5 years: a clusterrandomized, controlled trial. Circulation. 2014 Jan 21;129(3):321-329

11. Scott JA, Ng SY, Cobiac L. The relationship between breastfeeding and weight status in a national sample of Australian children and adolescents. BMC Public Health. 2012; 12:107

12. Li R, Fein SB, Grummer-Strawn LM. Do infants fed from bottles lack self-regulation of milk intake compared with directly breastfed infants? Pediatrics. 2010;125(6). Available at: www.pediatrics.org/cgi/content/ full/125/6/e1386

13. Mihrshahi S, Battistutta D, Magarey A, Daniels LA. Determinants of rapid weight gain during infancy: baseline results from the NOURISH randomised controlled trial. BMC Pediatr. 2011;11:99

14. Arenz $S$, Rückerl R, Koletzko B, von Kries R. Breast-feeding and childhood obesitya systematic review. Int J Obes Relat Metab Disord. 2004;28(10):1247-1256
15. Owen CG, Martin RM, Whincup PH, Smith GD, Cook DG. Effect of infant feeding on the risk of obesity across the life course: a quantitative review of published evidence. Pediat rics. 2005;115(5):1367-1377

16. Huh SY, Rifas-Shiman SL, Taveras EM, Oken E, Gillman MW. Timing of solid food introduction and risk of obesity in preschool-aged children. Pediatrics. 2011;127(3). Available at: www.pediatrics.org/cgi/content/full/ 127/3/e544

17. Grote V, Schiess SA, Closa-Monasterolo R, et al; European Childhood Obesity Trial Study Group. The introduction of solid food and growth in the first 2 y of life in formula-fed children: analysis of data from a European cohort study. Am J Clin Nutr. 2011;94(suppl 6):1785S-1793S

18. Grote V, Theurich M, Koletzko B. Do complementary feeding practices predict the later risk of obesity? Curr Opin Clin Nutr Metab Care. 2012;15(3):293-297

19. Ong KK. Early determinants of obesity. Endocr Dev. 2010;19:53-61

20. Gaffney KF, Kitsantas P, Cheema J. Clinical practice guidelines for feeding behaviors and weight-for-age at 12 months: a secondary analysis of the Infant Feeding Practices Study II. Worldviews Evid Based Nurs. 2012;9(4):234-242

21. Faith MS, Dennison BA, Edmunds LS, Stratton $\mathrm{HH}$. Fruit juice intake predicts increased adiposity gain in children from low-income families: weight status-by-environment interaction. Pediatrics. 2006;118(5):2066-2075 
22. DeBoer MD, Scharf RJ, Demmer RT. Sugarsweetened beverages and weight gain in 2- to 5-year-old children. Pediatrics. 2013; 132(3):413-420

23. Lumeng JC, Ozbeki TN, Appuǵliese DP, Kaciroti N, Corwyn RF, Bradley RH. Observed assertive and intrusive maternal feeding behaviors increase child adiposity. Am $J$ Clin Nutr. 2012;95(3):640-647

24. Thompson AL, Mendez MA, Borja JB, Adair LS, Zimmer CR, Bentley ME. Development and validation of the Infant Feeding Style Questionnaire. Appetite. 2009;53 (2):210-221

25. Bentley ME, Wasser HM, Creed-Kanashiro HM. Responsive feeding and child undernutrition in low- and middle-income countries. J Nutr. 2011;141(3):502-507

26. DiSantis KI, Hodges EA, Johnson SL, Fisher J0. The role of responsive feeding in overweight during infancy and toddlerhood: a systematic review. Int J Obes (Lond). 2011;35(4):480-492

27. Kimbro RT, Brooks-Gunn J, McLanahan S. Racial and ethnic differentials in overweight and obesity among 3-year-old children. Am J Public Health. 2007;97(2):298305

28. Manios Y, Kourlaba G, Kondaki K, Grammatikaki E, Anastasiadou A, Roma-Giannikou E. Obesity and television watching in preschoolers in Greece: the GENESIS study. Obesity (Silver Spring). 2009;17(11):20472053

29. Robinson TN. Reducing children's television viewing to prevent obesity: a randomized controlled trial. JAMA. 1999;282(16):15611567

30. Robinson TN, Matheson DM, Kraemer HC, et al. A randomized controlled trial of culturally tailored dance and reducing screen time to prevent weight gain in low-income African American girls: Stanford GEMS. Arch Pediatr Adolesc Med. 2010;164(11): 995-1004

31. Gortmaker SL, Must A, Sobol AM, Peterson K, Colditz GA, Dietz WH. Television viewing as a cause of increasing obesity among children in the United States, 1986-1990. Arch Pediatr Adolesc Med. 1996;150(4): 356-362

32. Crespo CJ, Smit E, Troiano RP, Bartlett SJ, Macera CA, Andersen RE. Television watching, energy intake, and obesity in US children: results from the third National Health and Nutrition Examination Survey, 19881994. Arch Pediatr Adolesc Med. 2001;155 (3):360-365

33. Jago R, Baranowski T, Baranowski JC, Thompson D, Greaves KA. BMI from 3-6 y of age is predicted by TV viewing and physical activity, not diet. Int J Obes (Lond). 2005;29 (6):557-564

34. Vangeepuram N, Mervish N, Galvez MP, Brenner B, Wolff MS. Dietary and physical activity behaviors of New York City children from different ethnic minority subgroups. Acad Pediatr. 2012;12(6):481-488

35. Schuster MA, Elliott MN, Kanouse DE, et al Racial and ethnic health disparities among fifth-graders in three cities. $N$ Engl $J$ Med. 2012;367 (8):735-745

36. Bremer AA, Byrd RS, Auinger P. Racial trends in sugar-sweetened beverage consumption among US adolescents: 19882004. Int J Adolesc Med Health. 2011;23(3): 279-286

37. Taveras EM, Gillman MW, Kleinman K, RichEdwards JW, Rifas-Shiman SL. Racial/ethnic differences in early-life risk factors for childhood obesity. Pediatrics. 2010;125(4): 686-695

38. Harris PA, Taylor R, Thielke R, Payne J, Gonzalez N, Conde JG. Research electronic data capture (REDCap) - a metadata-driven methodology and workflow process for providing translational research informatics support. J Biomed Inform. 2009;42 (2):377-381

39. Hediger ML, Overpeck MD, Ruan WJ, Troendle JF. Early infant feeding and growth status of US-born infants and children aged 4-71 mo: analyses from the third National Health and Nutrition Examination Survey, 1988-1994. Am J Clin Nutr. 2000;72 (1):159-167

40. Baker JL, Michaelsen KF, Rasmussen KM, Sørensen TI. Maternal prepregnant body mass index, duration of breastfeeding, and timing of complementary food introduction are associated with infant weight gain. $A m$ J Clin Nutr. 2004;80(6):1579-1588

41. Kavanagh KF, Cohen RJ, Heinig MJ, Dewey $K G$. Educational intervention to modify bottle-feeding behaviors among formulafeeding mothers in the WIC program: impact on infant formula intake and weight gain. J Nutr Educ Behav. 2008;40 (4) :244-250

42. Dennison BA, Edmunds LS, Stratton HH, Pruzek RM. Rapid infant weight gain predicts childhood overweight. Obesity (Silver Spring). 2006;14(3):491-499

43. Dennison BA, Erb TA, Jenkins PL. Television viewing and television in bedroom associated with overweight risk among lowincome preschool children. Pediatrics. 2002;109(6):1028-1035

44. Li R, Fein SB, Grummer-Strawn LM. Association of breastfeeding intensity and bottleemptying behaviors at early infancy with infants' risk for excess weight at late infancy. Pediatrics. 2008;122(suppl 2):S77S84

45. Brown A; Council on Communications and Media. Media use by children younger than 2 years. Pediatrics. 2011;128(5): 1040-1045

46. Laughlin J, Luerssen TG, Dias MS; Committee on Practice and Ambulatory Medicine, Section on Neurological Surgery. Prevention and management of positional skull deformities in infants. Pediatrics. 2011; 128(6): 1236-1241

47. McCullagh P. Regression models for ordinal data. J R Stat Soc, B. 1980;42:109-142

48. Harrell FE. Regression Modeling Strategies: With Applications to Linear Models, Logistic Regression, and Survival Analysis. New York, NY: Springer; 2001

49. Dixon B, Peña MM, Taveras EM. Lifecourse approach to racial/ethnic disparities in childhood obesity. Adv Nutr. 2012;3(1):73-82

50. Lapierre MA, Piotrowski JT, Linebarger DL. Background television in the homes of US children. Pediatrics. 2012;130(5):839-846

51. Rideout V, Hamel E. The Media Family: Electronic Media in the Lives of Infants, Toddlers, Preschoolers and Their Parents. Menlo Park, CA: Henry J. Kaiser Family Foundation; 2006

52. Thompson AL, Adair LS, Bentley ME. Maternal characteristics and perception of temperament associated with infant TV exposure. Pediatrics. 2013;131(2). Available at: www.pediatrics.org/cgi/content/full/131/ 2/e390

53. Brotanek JM, Halterman JS, Auinger $P$, Flores G, Weitzman M. Iron deficiency, prolonged bottle-feeding, and racial/ ethnic disparities in young children. Arch Pediatr Adolesc Med. 2005;159(11):10381042

54. Jones JR, Kogan MD, Singh GK, Dee DL, Grummer-Strawn LM. Factors associated with exclusive breastfeeding in the United States. Pediatrics. 2011;128(6): 1117-1125

55. Kumanyika SK. Environmental influences on childhood obesity: ethnic and cultural influences in context. Physiol Behav. 2008; 94(1):61-70

56. Ogbuanu CA, Probst J, Laditka SB, Liu J, Baek J, Glover S. Reasons why women do not initiate breastfeeding: a southeastern state study. Womens Health Issues. 2009;19 (4):268-278

57. Clarke PJ, O'Malley PM, Johnston LD, Schulenberg JE, Lantz P. Differential trends in weight-related health behaviors among American young adults by gender, race/ ethnicity, and socioeconomic status: 1984- 
2006. Am J Public Health. 2009;99(10): 1893-1901

58. Comstock RD, Castillo EM, Lindsay SP. Four-year review of the use of race and ethnicity in epidemiologic and public health research. Am J Epidemiol. 2004; 159(6):611-619
59. Ulmer C, McFadden B, Nerenz DR. Race, Ethnicity, and Language Data: Standardization for Health Care Quality Improvement. Washington, DC: National Academies Press; 2009

60. Suglia SF, Duarte CS, Chambers EC, BoyntonJarrett R. Cumulative social risk and obesity in early childhood. Pediatrics. 2012;
129(5). Available at: www.pediatrics.org/ cgi/content/full/129/5/e1173

61. Caprio S, Daniels SR, Drewnowski A, et al. Influence of race, ethnicity, and culture on childhood obesity: implications for prevention and treatment. Obesity (Silver Spring). 2008;16(12):2566-2577

(Continued from first page)

www.pediatrics.org/cgi/doi/10.1542/peds.2013-1326

doi:10.1542/peds.2013-1326

Accepted for publication Jan 13, 2014

Address correspondence to Eliana M. Perrin, MD, MPH, Associate Professor, Department of Pediatrics and Division of General Pediatrics and Adolescent Medicine, University of North Carolina-Chapel Hill School of Medicine, 231 MacNider Building, 333 South Columbia St, CB\#7225, Chapel Hill, NC 27599-7225. E-mail: eliana_perrin@med.unc.edu

PEDIATRICS (ISSN Numbers: Print, 0031-4005; Online, 1098-4275).

Copyright (C) 2014 by the American Academy of Pediatrics

FINANGIAL DISCLOSURE: The authors have indicated they have no financial relationships relevant to this article to disclose.

FUNDING: Supported with funding from the National Institutes of Health, Eunice Kennedy Shriver National Institute of Child Health and Human Development (R01 HD049794) and the National Institutes of Health, National Center for Advancing Translational Sciences (UL1TR000445, UL1TR000038, and UL1RR025747). Dr Yin is supported by a grant under the Robert Wood Johnson Foundation Physician Faculty Scholars Program and the Health Resources and Services Administration (12-191-1077-Academic Administrative Units in Primary Care). Dr Skinner is supported by a Building Interdisciplinary Research Careers in Women's Health career development award (K12 HD01441). Funded by the National Institutes of Health (NIH).

POTENTIAL CONFLICT OF INTEREST: The authors have indicated they have no potential conflicts of interest to disclose. 